\title{
Hyperpigmentation on the Head and Neck
}

Blake E. Vest, MD; Spyros M. Siscos, MD; Anand Rajpara, MD
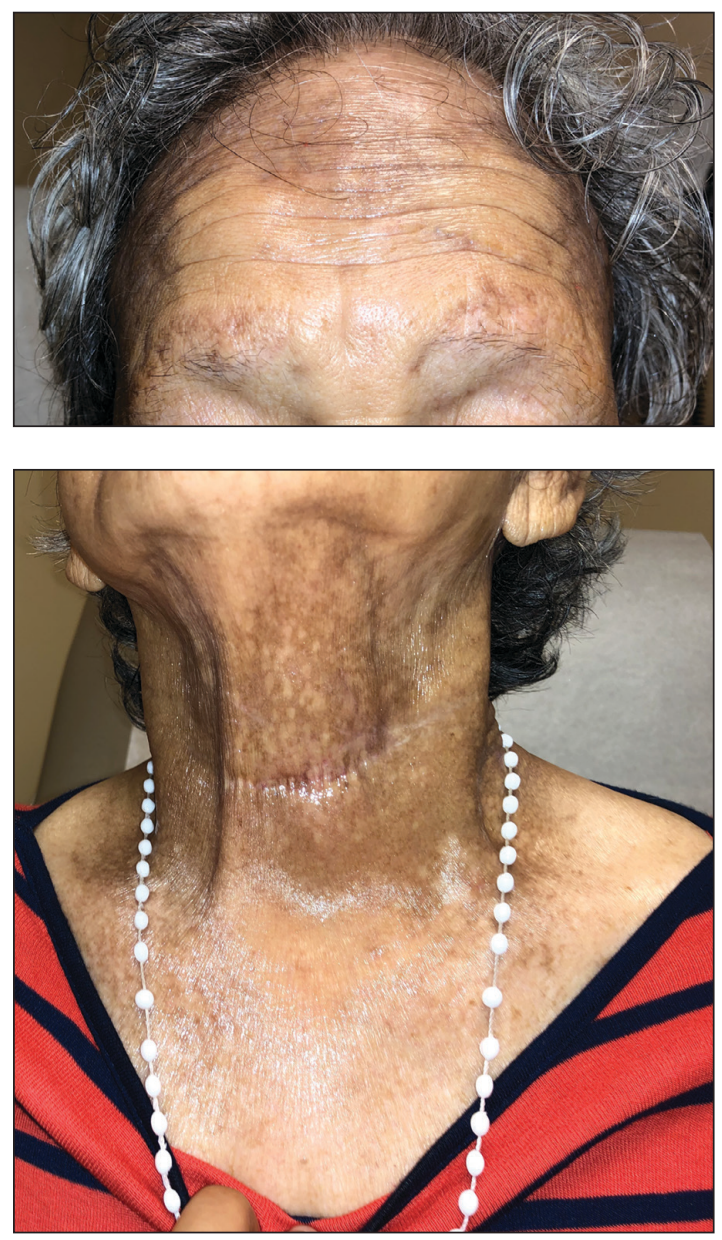

A 78-year-old Asian woman presented to the dermatology clinic with progressively worsening dark spots on the forehead and neck of 3 months' duration. She noted mild pruritis and hair loss involving the eyebrows and anterior scalp. Her medical history was notable for type 2 diabetes mellitus. She denied any new medical conditions or medications and had no prior history of similar symptoms. Physical examination showed hyperpigmented brown macules and patches on the forehead (top) and anterior neck (bottom) with sparing of the posterior neck and lower face. Alopecia with areas of perifollicular erythema and hyperpigmentation with reduced follicular openings were present on the eyebrows and anterior forehead. Two punch biopsies of head and neck lesions were performed.

\section{WHAT'S YOUR DIAGNOSIS?}

a. ashy dermatosis

b. frontal fibrosing alopecia overlapping with lichen planus pigmentosus

c. keratosis follicularis spinulosa decalvans

d. postinflammatory hyperpigmentation

e. pseudopelade of Brocq

\footnotetext{
Dr. Vest is from Southern Illinois University School of Medicine, Springfield. Drs. Siscos and Rajpara are from the Division of Dermatology, University of Kansas School of Medicine, Kansas City.

The authors report no conflict of interest.

Correspondence: Blake E. Vest, MD (bevest94@gmail.com). doi:10.12788/cutis.0226
} 


\section{THE DIAGNOSIS:}

\section{Frontal Fibrosing Alopecia Overlapping With Lichen Planus Pigmentosus}

M icroscopic examination revealed focal dermal pigmentation, papillary fibrosis, and epidermal atrophy. These clinical and histologic findings indicated a diagnosis of fully developed lichen planus pigmentosus (LPP) overlapping with frontal fibrosing alopecia (FFA). Other cases have demonstrated an association between LPP and FFA. ${ }^{1,2}$

Lichen planus pigmentosus is considered an uncommon variant of lichen planus, as it has similar histopathologic findings and occasional coexistence. ${ }^{3,4}$ It is characterized by hyperpigmented macules primarily located in sun-exposed and flexural areas of the skin. First described in India, ${ }^{5}$ this disease has a predilection for darker skin (Fitzpatrick skin types III-V), ${ }^{6,7}$ and it has been reported in other racial and ethnic groups including Latin Americans, Middle Eastern populations, Japanese, and Koreans., ${ }^{4,8}$ Typically, lesions initially appear as illdefined, blue-grey, round to oval macules that coalesce into hyperpigmented patches. Involvement most commonly begins at the forehead and temples, which are affected in nearly all patients. Infrequently, LPP can be generalized or affect the oral mucosa; involvement of the palms, soles, and nails does not occur. Patients may be asymptomatic, but some experience mild pruritus and burning. The disease course is chronic and insidious, with new lesions appearing over time and old lesions progressively darkening and expanding. ${ }^{6,7,9}$

Although the pathogenesis of LPP is unknown, several exposures have been implicated, such as amla oil, mustard oil, henna, hair dye, and environmental pollutants. ${ }^{7}$ Because lesions characteristically occur in sunexposed areas, UV light also may be involved. In addition, studies have suggested that LPP is associated with endocrinopathies such as diabetes mellitus and dyslipidemias, as in our patient, as well as autoimmune conditions such as vitiligo and systemic lupus erythematosus. ${ }^{10,11}$

Histopathologic findings are characterized by vacuolar degeneration of the basal layer in the epidermis as well as perivascular lymphohistiocytic infiltration and the presence of melanophages in the dermis. ${ }^{3,9}$ Lichen planus pigmentosus is difficult to treat, as no consistently effective modality has been established. Topical tacrolimus, topical corticosteroids, oral retinoids, lasers, and sun protection have been implemented with underwhelming results. ${ }^{12}$

Frontal fibrosing alopecia is a variant of lichen planopilaris that predominantly affects postmenopausal women and presents with frontotemporal hair loss in a bandlike distribution. ${ }^{5,13}$ Both terminal and vellus hairs are affected. Involvement of multiple hair-bearing sites of the skin have been reported, including the entire scalp, eyebrows, and eyelashes. Affected areas may display hypopigmentation and be accompanied by pruritus and trichodynia. ${ }^{14,15}$ The pathogenesis currently is under investigation, with studies demonstrating autoimmune, genetic, and possibly even endocrine predispositions. ${ }^{16-18}$ Biopsies of lesions are indistinguishable from lichen planopilaris, which shows follicular tymphocytic infiltration, perifollicular fibrosis, interface dermatitis of the follicular infundibulum and isthmus, and vertical fibrous tracks. ${ }^{5}$ Patients with FFA have demonstrated variable responses to treatments, with one study showing improvement with oral finasteride or dutasteride. ${ }^{14}$ Topical and intralesional corticosteroids have yielded suboptimal effects. Other modalities include hydroxychloroquine and mycophenolate mofetil. 15,19

Co-occurrence of LPP and FFA primarily is seen in postmenopausal women with darker skin, ${ }^{14,15}$ as in our patient, though premenopausal cases have been reported. Lichen planus pigmentosus may serve as a harbinger in most patients. ${ }^{1,2}$ In a similar fashion, our patient presented with hyperpigmented macular lesions prior to the onset of frontotemporal hair loss.

Our patient was started on finasteride $2.5 \mathrm{mg}$ daily, minoxidil foam $5 \%$, clobetasol solution $0.05 \%$, triamcinolone ointment $0.1 \%$, and hydrocortisone ointment $2.5 \%$. She was instructed to commence treatment and follow up in 6 months.

The differential diagnosis includes dermatologic conditions that mimic both LPP and FFA. Postinflammatory hyperpigmentation and fixed drug reaction were unlikely based on the patient's history. The lesions of ashy dermatosis are characteristically gray erythematous macules on the trunk and limbs. Riehl melanosis is a rare pigmented contact dermatitis that is associated with a history of repeated contact with sensitizing allergens. Although Hori nevus is characterized by small, blue-gray or brown macules on the face, lesions predominantly occur on the bony prominences of the cheeks. Melasma also presents with dark to gray macules that affect the face and less commonly the neck, as in our patient. ${ }^{2}$

Early discoid lupus erythematosus presents with round erythematous plaques with overlying scale extending into the hair follicles. In pseudopalade of Brocq, an idiopathic cicatricial alopecia, lesions typically are flesh colored. Biopsy also shows epidermal atrophy with additional dermal sclerosis and fibrosis. Folliculitis decalvans is a scarring form of alopecia associated with erythema and pustules, findings that were not present in our patient. Keratosis follicularis spinulosa decalvans is a 
rare, $\mathrm{X}$-linked inherited ichthyosis manifesting as scarring alopecia with follicular depressions and papules on the scalp in younger males. Photophobia and other manifestations may be present. Alopecia mucinosa is a nonscarring alopecia with grouped follicular erythematous patches or plaques. Mucin sometimes can be squeezed from affected areas, and histopathologic examination shows mucin accumulation. ${ }^{4}$

\section{REFERENCES}

1. Dlova NC. Frontal fibrosing alopecia and lichen planus pigmentosus: is there a link? Br J Dermatol. 2013;168:439-442.

2. Pirmez R, Duque-Estrada B, Donati A, et al. Clinical and dermoscopic features of lichen planus pigmentosus in 37 patients with frontal fibrosing alopecia. Br J Dermatol. 2016;175:1387-1390.

3. Rieder E, Kaplan J, Kamino H, et al. Lichen planus pigmentosus. Dermatol Online J. 2013;19:20713.

4. Kashima A, Tajiri A, Yamashita A, et al. Two Japanese cases of lichen planus pigmentosus-inversus. Int J Dermatol. 2007;46:740-742.

5. Bhutani L, Bedi T, Pandhi R. Lichen planus pigmentosus. Dermatologica. 1974;149:43-50.

6. Ross EK, Tan E, Shapiro J. Update on primary cicatricial alopecias. J Am Acad Dermatol. 2005;53:1-37.

7. Kanwa AJ, Dogra S, Handa S, et al. A study of 124 Indian patients with lichen planus pigmentosus. Clin Exp Dermatol. 2003;28:481-485.

8. Al-Mutairi N, El-Khalawany M. Clinicopathological characteristics of lichen planus pigmentosus and its response to tacrolimus ointment: an open label, non-randomized, prospective study. J Eur Acad Dermatol Venereol. 2010;24:535-540.
9. Vega ME, Waxtein L, Arenas R, et al. Ashy dermatosis and lichen planus pigmentosus: a clinicopathologic study of 31 cases. Int J Dermatol. 1992;31:90-94.

10. Robles-Méndez JC, Rizo-Frías P, Herz-Ruelas ME, et al. Lichen planus pigmentosus and its variants: review and update. Int J Dermatol. 2018;57:505-514

11. Torres J, Guadalupe A, Reyes E, et al. Lichen planus pigmentosus in patients with endocrinopathies and hepatitis C. J Am Acad Dermatol. 2013;68:AB139

12. Kim JE, Won $\mathrm{CH}$, Chang $\mathrm{S}$, et al. Linear lichen planus pigmentosus of the forehead treated by neodymium:yttrium-aluminum-garnet laser and topical tacrolimus. J Dermatol. 2012;39:189-191.

13. Kossard S. Postmenopausal frontal fibrosing alopecia: scarring alopecia in a pattern distribution. Arch Dermatol. 1994;130:770-774.

14. Vano-Galvan S, Molina-Ruiz AM, Serrano-Falcon C, et al. Frontal fibrosing alopecia: a multicenter review of 355 patients. J Am Acad Dermatol. 2014;70:670-678.

15. MacDonald A, Clark C, Holmes S. Frontal fibrosing alopecia: a review of 60 cases. J Am Acad Dermatol. 2012;67:955-961.

16. Harries MI, Meyer K, Chaudhry I, et al. Lichen planopilaris is characterized by immune privilege collapse of the hair follicle's epithelial stem cell niche. J Pathol. 2013;231:236-247.

17. Karnik P, Tekeste Z, McCormick TS, et al. Hair follicle stem cell-specific PPARgamma deletion causes scarring alopecia. J Invest Dermatol. 2009; 129:1243-1257.

18. Rodriguez-Bayona B, Ruchaud S, Rodriguez C, et al. Autoantibodies against the chromosomal passenger protein INCENP found in a patient with Graham Little-Piccardi-Lassueur syndrome. J Autoimmune Dis. 2007:4:1.

19. Rácz E, Gho C, Moorman PW, et al. Treatment of frontal fibrosing alopecia and lichen planopilaris: a systematic review. J Eur Acad Dermatol Venereol. 2013;27:1461-1470. 